\title{
ATM regulates ATR chromatin loading in response to DNA double-strand breaks
}

\author{
Myriam Cuadrado, Barbara Martinez-Pastor, Matilde Murga, Luis I. Toledo, \\ Paula Gutierrez-Martinez, Eva Lopez, and Oscar Fernandez-Capetillo
}

Genomic Instability Group, Spanish National Cancer Center, Madrid 28029, Spain

DNA double-strand breaks (DSBs) are among the most deleterious lesions that can challenge genomic integrity. Concomitant to the repair of the breaks, a rapid signaling cascade must be coordinated at the lesion site that leads to the activation of cell cycle checkpoints and/or apoptosis. In this context, ataxia telangiectasia mutated (ATM) and ATM and Rad-3-related (ATR) protein kinases are the earliest signaling molecules that are known to initiate the transduction cascade at damage sites. The current model places ATM and ATR in separate molecular routes that orchestrate distinct pathways of the checkpoint responses. Whereas ATM signals DSBs arising from ionizing radiation (IR) through a Chk2dependent pathway, ATR is activated in a variety of replication-linked DSBs and leads to activation of the checkpoints in a Chk1 kinase-dependent manner. However, activation of the G2/M checkpoint in response to IR escapes this accepted paradigm because it is dependent on both ATM and ATR but independent of Chk2. Our data provides an explanation for this observation and places ATM activity upstream of ATR recruitment to IR-damaged chromatin. These data provide experimental evidence of an active cross talk between ATM and ATR signaling pathways in response to DNA damage.

CORRESPONDENCE

Oscar Fernandez-Capetillo: ofernandez@cnio.es
Cell cycle checkpoints coordinate a timely progression throughout the cell cycle that is fundamental to ensure that critical events such as DNA replication and chromosome segregation are completed accurately before cell division takes place (1). The integrity of the process is verified both at the G1/S and G2/M transition boundaries as well as during $\mathrm{S}$ phase, with the resultant monitoring by a specific checkpoint pathway in each case. In response to DNA double-strand breaks (DSBs) and together with the assembly of the DNA repair complexes, cell cycle checkpoints are activated that either delay cell cycle progression until the breaks are repaired or promote apoptosis, depending on the cell type and/or the amount of damage (2). Accordingly, the accumulation of genomic aberrations caused by the loss of cell cycle checkpoints has classically been interpreted as one of the motors that enables the increased mutation rate necessary for cancer development (3). Moreover, recent data has showed that activation of the DNA damage

M. Cuadrado and B. Martinez-Pastor contributed equally to this paper.

The online version of this article contains supplemental material. checkpoint is also triggered by oncogenic stress and is thus a conserved feature of early precancerous lesions $(4,5)$. Besides cancer, a proper response to DNA breaks is also seminal to handle the lesions generated during programmed recombination processes such as in meiosis or during $\mathrm{V}(\mathrm{D}) \mathrm{J}$ and class switch recombination reactions in lymphoid cells.

At the molecular level, activation of the DNA damage checkpoints is based on a phosphorylation cascade that starts with the activation of certain members of the PIKK (phosphatidyl-inositol 3 kinaselike protein kinase) family at the site of the lesion (6). Among the six PIKK proteins expressed in mammalian cells ataxia telangiectasia (AT) mutated (ATM) and ATM and Rad-3 related (ATR) are the two key members in triggering the DNA damage transduction cascade and coordinating individual checkpoints. Although a third PIKK (DNA-PKcs) might also phosphorylate certain DNA damage response proteins in response to DSBs, its action seems more restricted to the break site and not in coordinating a global cellular response (7). Our current understanding places ATM and ATR in two separate and alternate pathways of checkpoint activation that 
A

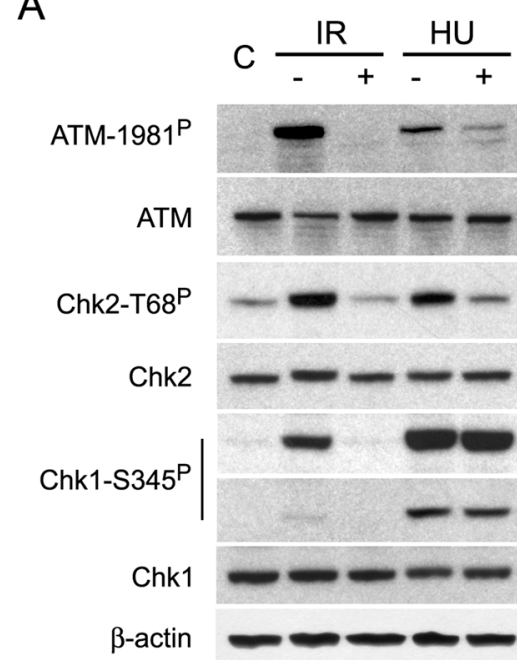

B

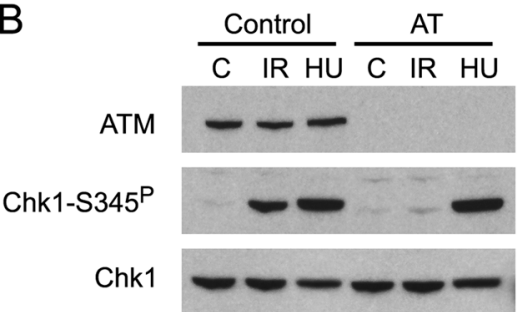

Figure 1. Analysis of ATM, Chk2, and Chk1 activation in response to DNA damage. (A) Western blot detection of ATM-1981 ${ }^{\text {, ATM }}$, Chk2T68 ${ }^{P}$, Chk2, Chk1-S345P, and Chk1 in Raji human lymphoblastoid cells exposed to DNA-damaging conditions. Where indicated, ATM activity was inhibited by a previous 15-min treatment with $20 \mu \mathrm{M}$ wortmannin (WM). (B) Evaluation of Chk1 phosphorylation dynamics in control and AT human cells in response to DNA damage. (C) Western blot analysis of ATR, Chk1-S345 P, Chk1, ATM-1981P, and ATM levels in extracts from control (ATRwt) and ATR hypomorphic

converge at the cyclin-dependent kinase (CDK) activity inhibition step. On one hand, the response to DSBs such as those generated by ionizing radiation (IR) is triggered through ATM, and the signal is further amplified by ATM-dependent activation of the downstream kinase Chk2. On the other hand, an analogue pathway that works through ATR and the Chk2-relative Chk1 is accountable for the response to DSBs arising at replication forks. However, a detailed analysis of the distinct effects of ATM, ATR, Chk2, and Chk1 deficiencies on checkpoint control challenges this simplistic view and implies that novel interactions among these kinases are necessary to explain the observed genetic requirements. Although ATM deficiency leads to the loss of control of all G1/S, intra-S, and G2/M checkpoints in response to IR (8),
C
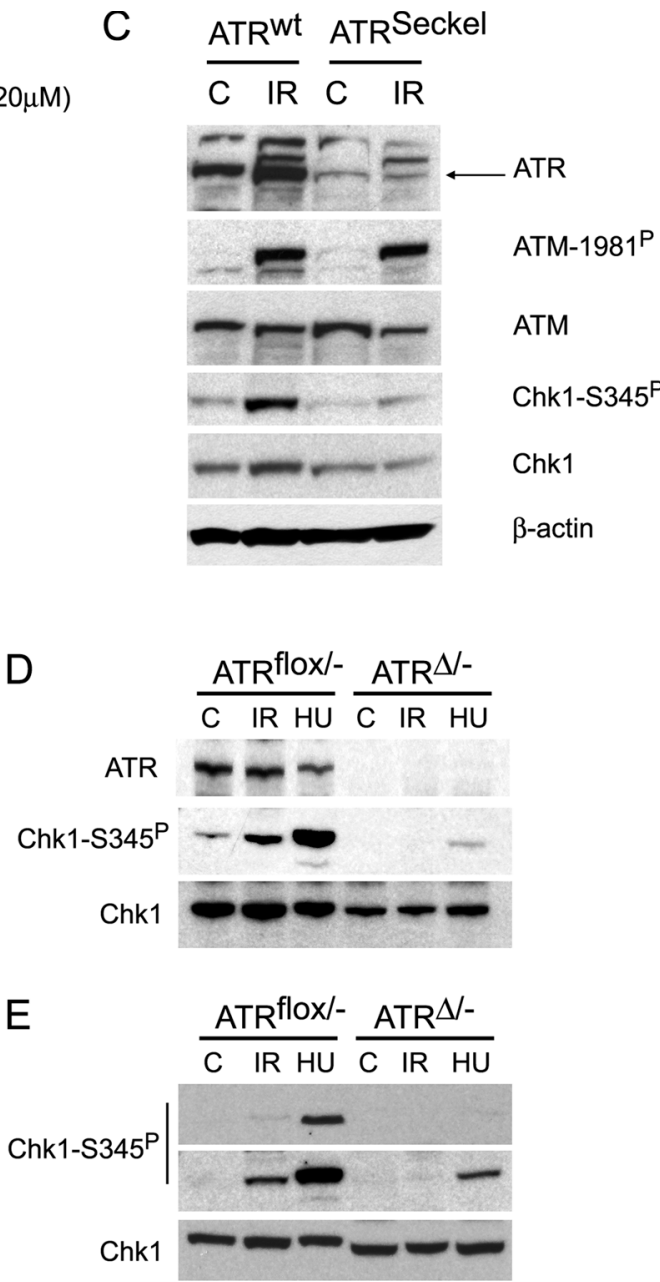

(ATR ${ }^{\text {Seckel) }}$ human cells irradiated with $20 \mathrm{~Gy}$ (or mock irradiated [C]). $\beta$-actin levels were evaluated as a loading control. (D) Analysis of Chk1 phosphorylation in ATR ${ }^{\text {flox }}$ - cells $48 \mathrm{~h}$ after infection with Cre-expressing adenovirus (ATR ${ }^{\Delta /-}$ ) or mock infection (ATR ${ }^{\text {floxl--}}$ ). (E) The same analysis as in $D$, in which the loading was normalized to eliminate differences in total Chk1 levels. In all cases, exponentially growing cells were irradiated with $20 \mathrm{~Gy}$, and the extracts were performed $45 \mathrm{~min}$ after irradiation (IR) or $2 \mathrm{~h}$ after exposure to $2 \mathrm{mM}$ hydroxyurea (HU).

Chk2 knockout cells have no obvious defect in intra-S or G2/M checkpoint activation and show only a modest impairment of the $\mathrm{G} 1 / \mathrm{S}$ responses $(9,10)$. In contrast, although the ATR-Chk1 pathway is generally associated exclusively with replication-associated DSBs, both kinases are required for the IR-induced G2/M checkpoint $(11,12)$. In addition, phosphorylation of Chk1 is also detected in cells exposed to IR, but the interpretation of this result has classically been linked to the loss of specificity of ATM at high amounts of damage. In contrast with this view, we now have data showing that this Chk1 phosphorylation in response to IR is the outcome of a fine regulation that derives from the coordinated action of both ATM and ATR. Our results demonstrate that in response to IR, ATR is loaded onto chromatin 


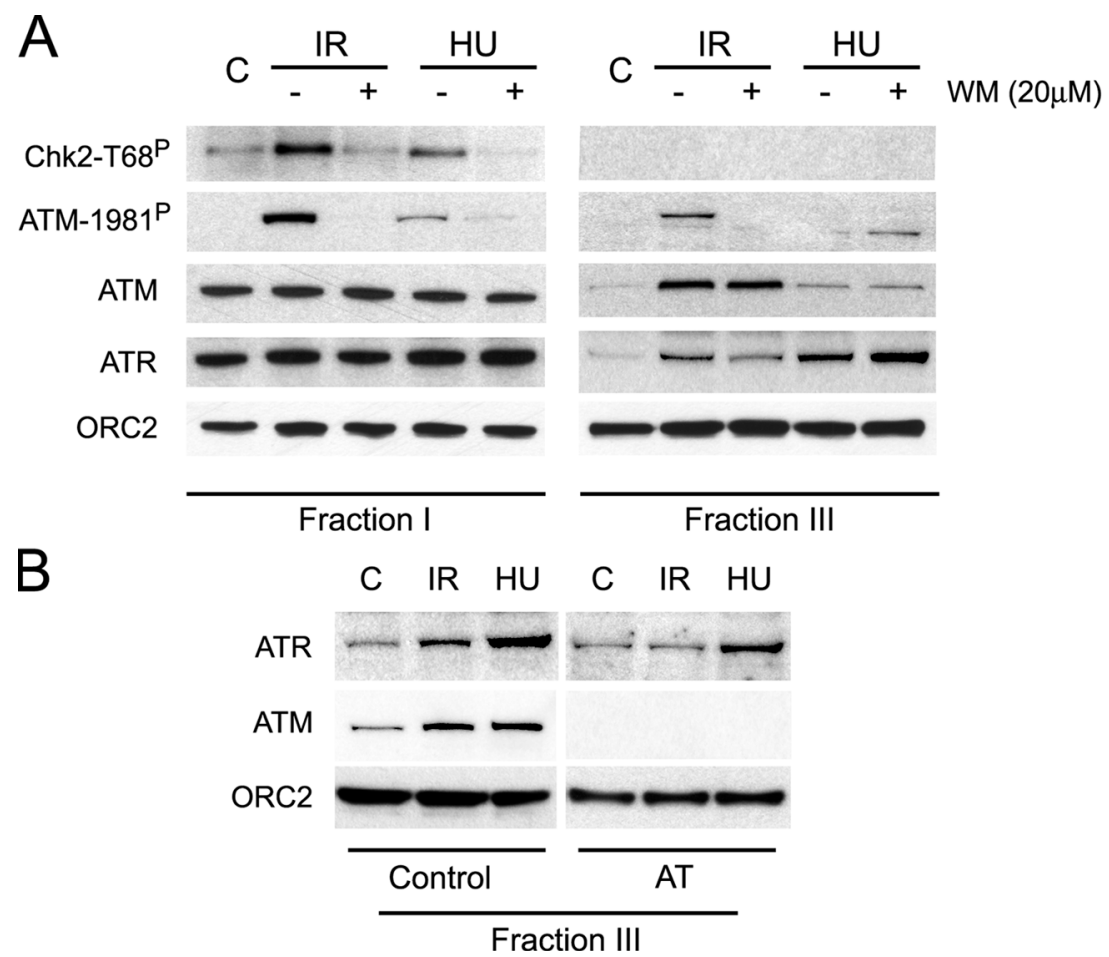

Figure 2. Nuclear retention of ATM and ATR in response to DSBs. (A) The association of ATM and ATR with chromatin in the various treatments was evaluated in Raji cells by a serial fractionation protocol (see Materials and methods). Fraction I (cytoplasmic) and fraction III (chromatin bound) are shown from the same extracts. ATM-1981P and Chk2-T68 ${ }^{P}$ levels are shown as controls of the wortmannin inhibitory effect. No detectable Chk2-T68 ${ }^{P}$ is bound to the chromatin with this protocol. The levels of the replication factor ORC2 are shown as a loading control in both cases because it is present both in the cytoplasmic as well as in a nuclear fraction. Notably, the association of ORC2 with chromatin does not change in response to DNA damage. The figure illustrates the common observation of a set of experiments $(n=5)$. (B) Chromatin-bound fraction analysis in control and AT human cell lines. Treatments: $C_{\text {, con- }}$ trol; IR, 20 Gy irradiation for 45 min; HU, 2 mM hydroxyurea for $2 \mathrm{~h}$. in an ATM-dependent manner that, for the first time, demonstrates an active cross talk between ATM and ATR in the DNA damage response. Finally, the development of a new flow cytometry-based detection assay for DNA damage signaling events has allowed us to discover that IR-induced Chk1 phosphorylation is restricted to late $\mathrm{S}$ and $\mathrm{G} 2$ cells, thus giving us a molecular framework to understand the series of events that govern the activation of the G2/M checkpoint.

\section{RESULTS AND DISCUSSION \\ IR-induced Chk1 phosphorylation is dependent on ATM and ATR}

To determine the molecular requirements that are responsible for Chk1 phosphorylation in response to IR or to reagents that generate DSBs at the replication forks, we evaluated Chk1 phosphorylation at S345 by Western blotting in cells exposed to IR or hydroxyurea (HU). The experiment was carried in the presence or absence of the PIKK inhibitor wortmannin at concentrations that have been reported to inhibit ATM but not ATR activity (Fig. 1 A; reference 13). The specificity of the inhibitor was validated by evaluating the levels of Chk2-T68 phosphorylation in the same conditions, which are strictly dependent on $\operatorname{ATM}(14,15)$. In addition, the levels of ATM-1981 ${ }^{\mathrm{P}}$ were also analyzed in our experi- ments and served as a hallmark of ATM activation status (16). The behavior of Chk2 phosphorylation was consistent with the current models and was found to be ATM dependent regardless of the DNA-damaging source. However, whereas Chk1-S345 phosphorylation is not dependent on ATM activity in cells exposed to HU or aphidicolin (Fig. S1, available at http://www.jem.org/cgi/content/full/jem.20051923/ DC1), an event controlled primarily by ATR (12), the phosphorylation of Chk1 in response to IR was found to be abrogated in cells pretreated with wortmannin. In agreement with this data, a similar regulation of Chk1 phosphorylation dynamics was observed in human ATM-deficient cells obtained from patients of AT (Fig. 1 B). Analogous results were obtained with two additional AT cell lines (not depicted). In summary, these data imply that ATM activity is upstream of Chk1 phosphorylation in response to IR but not replicationlinked DSBs.

Because ATR and not ATM is responsible for Chk1S345 phosphorylation in response to stalled replication forks (12), we decided to evaluate the effect of ATR deficiency in the Chk1 phosphorylation observed in response to radiation. To do this, IR-induced Chk1-345 ${ }^{\mathrm{P}}$ levels were analyzed in lymphoblastoid cells derived from patients of a human hereditable disease known as the Seckel syndrome, which is 
A
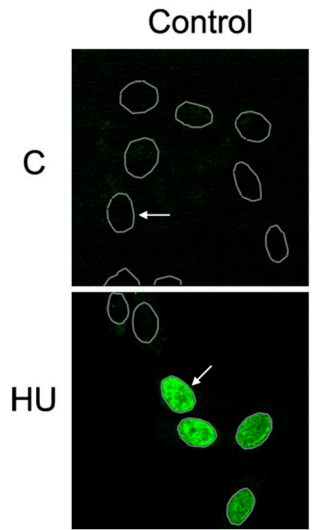

IR

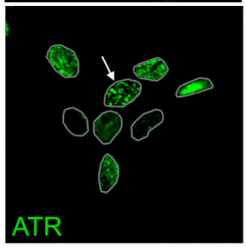

AT
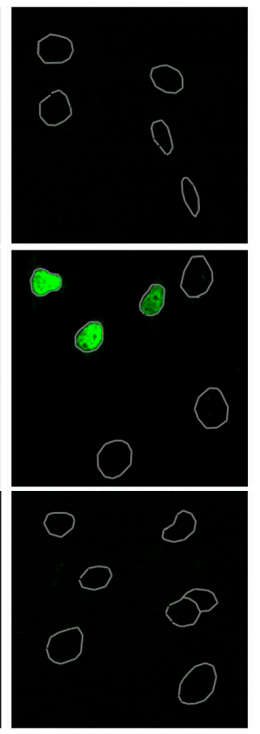

C

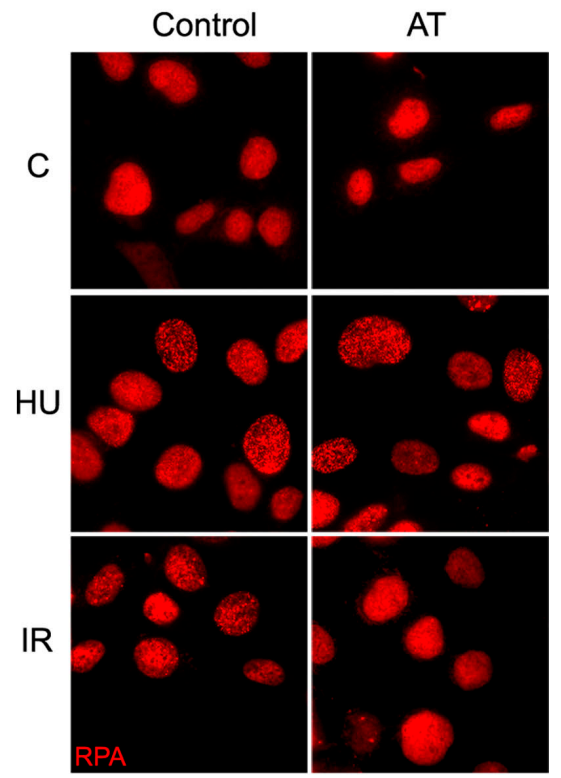

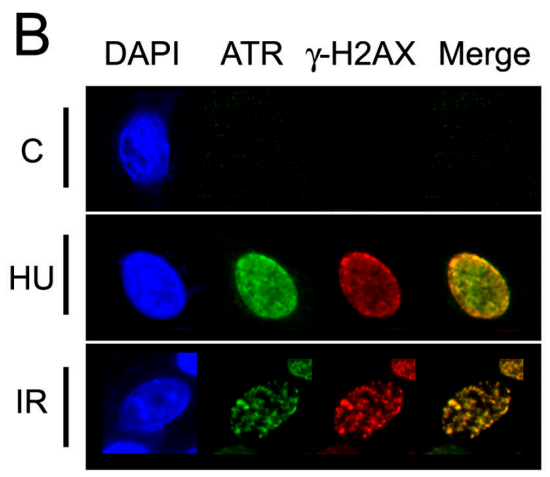

Figure 3. Analysis of RPA and ATR localization in response to DNA damage. (A) ATR immunostaining in control and AT cells exposed to DNAdamaging conditions. Because most of the ATR pool is not bound to chromatin in the absence of damage and the in situ extraction eliminates this fraction, a gray mask based on the DAPI channel is drawn that indicates the presence of nuclei in each field. (B) Images of ATR and $\gamma-\mathrm{H} 2 \mathrm{AX}$ signals in individual cells selected from A (arrows). (C) RPA immunostaining in control and AT cells. Equivalent to the biochemistry, exponentially growing cells were irradiated with $20 \mathrm{~Gy}$, and the cells were processed $45 \mathrm{~min}$ after irradiation (IR) or $2 \mathrm{~h}$ after exposure to $2 \mathrm{mM}$ hydroxyurea (HU). associated with a dramatic reduction in ATR levels as a result of a synonymous mutation that alters the splicing efficiency of the transcript (Fig. 1 C; reference 17). Whereas IR-induced Chk1-S345 ${ }^{\text {P }}$ levels were readily detected in the corresponding control line, almost no detectable signal was observed in ATR-Seckel cells. ATR-dependent phosphorylation of Chk1 in response to IR has also been observed in mouse embryo fibroblasts harboring conditional ATR alleles (11). To evaluate whether the Chk1 phosphorylation defect is not directly dependent on ATR and is caused by an inefficient activation of ATM in ATR-Seckel cells, IR-induced ATM$1981^{\mathrm{P}}$ levels were analyzed in both cell lines. Notably, ATM phosphorylation is not affected by the diminished levels of ATR found in ATR-Seckel cells, demonstrating that the ATR-dependent Chk1-S345 phosphorylation induced by
IR occurs downstream of ATM activation. To avoid variability that might have arisen during the prolonged passages of the different cell lines, we evaluated the activation of Chk1 in a previously published human cell line that is susceptible to ATR deletion upon infection with a Cre recombinase-

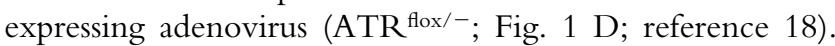
Interestingly, in both experimental systems, we noted that ATR depletion leads to a concomitant decrease in Chk1 levels. Because, as reported previously (18), no differences in cell cycle distribution were observed between $\mathrm{ATR}^{\text {flox/- }}$ and $\mathrm{ATR}^{\Delta /-}$ cells in the cells used for our biochemical analyses, this observation might be indicative of an indirect role for ATR in the regulation of Chk1 levels as part of triggering the DNA damage response. To avoid these differences and to directly evaluate the impact of ATR deficiency in Chk1 
phosphorylation, we normalized the loading by adjusting Chk1 levels between both cell lines (Fig. 1 E). The observations made in this conditional system are equivalent to the results obtained with Seckel cells and further demonstrate that Chk1 phosphorylation is controlled by ATR regardless of the type of damage. Altogether, our data demonstrates that whereas Chk1 activation in response to DSBs arising at stalled replication forks is strictly dependent on ATR, the phosphorylation of Chk1 in response to IR is coordinately regulated by a sequential ATM $\rightarrow$ ATR-dependent pathway.

\section{ATM regulates chromatin loading of ATR in response to DSBs}

The aforementioned biochemical data suggest that ATR works downstream of ATM in the regulation of Chk1 after exposure to IR. Given that ATM is upstream of the accumulation of several DSB repair/signaling proteins at damage sites, one potential explanation for our findings might occur if ATM is also involved in the redistribution of ATR onto damaged DNA. To evaluate this possibility, we first analyzed the chromatin loading of ATM and ATR in response to DNA damage by a nuclear fractionation technique (Fig. 2; reference 19). As reported, exposure to IR induced the chromatin retention of a fraction of the ATM pool. Notably, whereas wortmannin pretreatment eliminated IR-induced ATM-1981 ${ }^{\mathrm{P}}$ foci (not depicted), it did not affect the rapid chromatin loading of ATM in the same conditions, placing the global chromatin recruitment detected by this biochemical assay upstream of the formation of foci. HU treatment also induces a noticeable retention of ATM in the chromatin fraction, which was also unaffected upon ATM inhibition. From the simultaneous analysis of ATR recruitment to damaged chromatin, several conclusions can be obtained. First, extracts from HU and, to a lesser extent, IR triggered chromatin loading of ATR, demonstrating that the global chromatin retention in response to DNA damage previously observed for ATM is a conserved feature among early damage sensors. Strikingly, whereas the chromatin loading of ATR in response to HU was not diminished by ATM inactivation, IR-induced chromatin retention of ATR was severely abrogated in wortmannin-pretreated cells. Because the chromatin fractionation assay was performed $45 \mathrm{~min}$ after irradiation, no changes in cell cycle distribution are observed that could account for the differences in ATR loading (Fig. S2, available at http://www.jem.org/cgi/ content/full/jem.20051923/DC1). Finally, and consistent with our inhibition data, the analysis of chromatin loading of ATR onto damaged chromatin in AT cells demonstrated that whereas ATR is loaded into chromatin in HU-treated cells, no IR-induced retention of ATR is present in the absence of ATM (Fig. 2 B).

Equivalent to the biochemical fractionation technique used in Fig. 2, a protocol based on an in situ detergent preextraction of the nucleoplasmic fraction allows for the microscopical evaluation of chromatin-bound proteins in response to damage (20). Evaluation of ATR in AT and correspond-

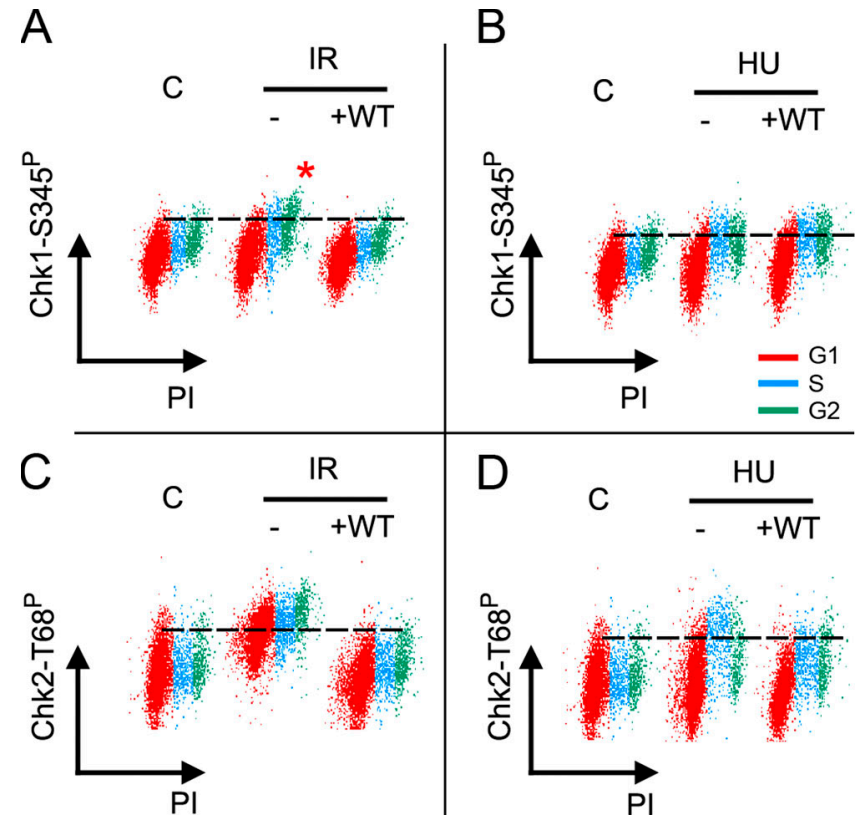

Figure 4. Flow cytometry analysis of Chk1 and Chk2 phosphorylation during the cell cycle. An aliquot of the same Raji cells that were used for Western blotting (Fig. 1) or chromatin fractionation (Fig. 2) was analyzed in parallel with multiparametric flow cytometry for DNA content with propidium iodide and for Chk1-S345 (A and B) or Chk2-T68 ( $\mathrm{C}$ and D) levels with the corresponding specific antibodies (see Materials and methods). Note the distinct increase in Chk1 phosphorylation in irradiated $\mathrm{G} 2$ cells (red asterisk). Dashed lines are incorporated in each case to illustrate the phosphorylation dynamics. Doses used: $20 \mathrm{~Gy} I \mathrm{R} ; 2 \mathrm{mM} \mathrm{HU}$,

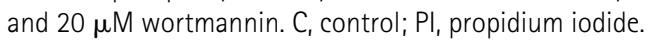

ing control cell lines by these means confirmed that ATR is loaded onto chromatin in an ATM-dependent manner in response to IR (Fig. 3 A). In agreement with our previous data, HU-induced ATR loading was not affected by ATM status. Moreover, HU-induced loading of ATR was significantly higher than that obtained in response to IR and showed a pan-nuclear staining that contrasted with the foci-like pattern observed in irradiated cells. Where detectable, ATR signal colocalized with $\gamma$-H2AX, suggesting that the difference in the staining pattern between IR- and HU-treated cells reflects a global generation of DSBs in replicating cells in contrast with the localized damage generated by IR (Fig. 3 B). The analysis of RPA (replication protein A) localization showed that whereas RPA is chromatin bound regardless of DNA damage, it relocalizes into discrete foci upon exposure to IR or HU. Remarkably, RPA localization to DSBs showed the same ATM dependency as ATR, which provides evidence that ATM regulation of ATR recruitment is mediated at the earliest steps of activation of the ATR-Chk1 pathway (Fig. $3 \mathrm{C}$ ). In summary, our data demonstrate that IR-induced chromatin loading of ATR is dependent on ATM activity and, thus, places ATM upstream of the ATR-Chk1 signaling pathway in the checkpoint responses initiated by radiation damage. 


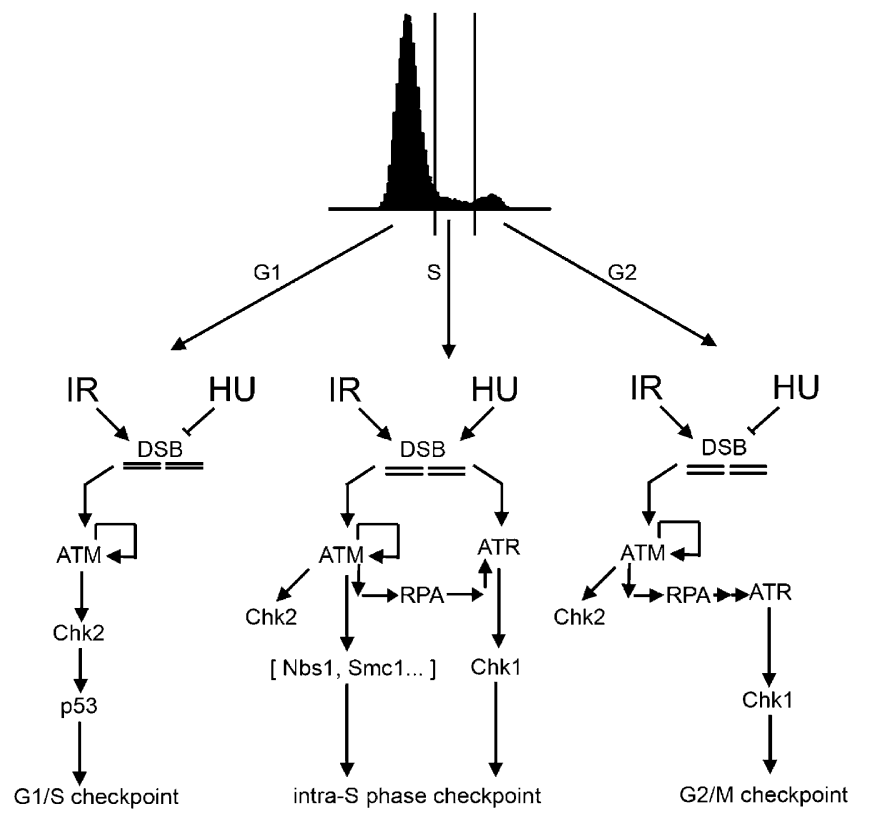

Figure 5. Interactions between ATM, ATR, Chk2, and Chk1 in the DNA damage response. The diagram illustrates the molecular determinants that control the responses to IR and $\mathrm{HU}$ initiated in the different stages of the cell cycle. Whereas ATR and Chk1 activities in checkpoint signaling are restricted to cells beyond S phase, ATM activity is necessary throughout the cell cycle.

\section{Cell cycle-specific phosphorylation of Chk1 and Chk2 in response to DNA damage}

In contrast to ATM, cell cycle checkpoint activities of ATR and Chk1 are restricted to the S and G2 phases of the cell cycle $(11,12,21)$. Interestingly, IR-induced ATR and RPA foci are only detected in a fraction of the cells, suggesting that activation of this pathway could be constrained to certain stages of the cell cycle. To evaluate this possibility and taking advantage of a recent method that optimized the detection of phosphoepitopes by cytometry (22), we established a new flow cytometry-based assay that allowed us to analyze the levels of Chk1 and Chk2 phosphorylation in each stage of the cell cycle. The results illustrated in Fig. 4 gave us a comprehensive picture of the cell cycle-dependent Chk1 and Chk2 phosphorylation profiles in response to DNA damage. Whereas an increase in Chk2-T68 $8^{\mathrm{P}}$ levels was detected in all stages of the cell cycle in response to IR, this increase was restricted to the $\mathrm{S}$ phase in HU-treated cells. This behavior is expected because in contrast to the unbiased nature of IR, the capacity of HU to generate DSBs is only present in replicating cells. Moreover (and consistent with the Western blot data), both IR- and HU-induced Chk2 phosphorylation were abrogated in cells pretreated with ATM-inhibiting doses of wortmannin. Thus, ATM-dependent Chk2 phosphorylation is triggered whenever a DSB is detected, regardless of the damaging source or the cell cycle stage. The analysis of Chk1-S345 phosphorylation dynamics by the same means produced a clarifying result. Similar to Chk2-T68
HU-induced Chk1 phosphorylation was only detected in replicating cells. Surprisingly, a similar behavior was found in response to IR, with the remarkable difference that Chk1 phosphorylation was most prominent in irradiated G2 cells. Furthermore (and consistent with the biochemical data presented in Fig. 1), Chk1 phosphorylation in S-G2 was abrogated in the absence of ATM activity. In summary, these results provide a direct explanation for the restricted role of Chk1 to S and G2 phases of the cell cycle in response to IR and present a comprehensive view of ATM and ATR activities throughout the cell cycle.

\section{A new model for ATM/ATR/Chk1 functions in response to DSBs}

The current data suggests a new model for the control of IRinduced checkpoint signaling pathways for the different stages of the cell cycle depicted in Fig. 5. In response to IR, ATM phosphorylates Chk2 throughout the cell cycle, which might particularly enhance the regulation of the G1 checkpoint through regulating p53 phosphorylation status. In $\mathrm{S}$ phase, both ATM and ATR coordinate checkpoint activities. On one hand, in response to reagents that promote stalled replication forks such as $\mathrm{HU}$ or aphidicolin, an ATR-Chk1 cascade is effective that is not dependent on ATM activity. Notably, a significantly higher loading of ATR onto the chromatin of HU-treated cells is detected in the absence of ATM activity (Fig. 2 A). This finding is in agreement with our model, and because HU triggers both ATM and ATR activities through independent pathways, it could be the outcome of compensatory loading of ATR in the absence of ATM. On the other hand, IR-induced ATM-dependent checkpoint signaling in $\mathrm{S}$ phase is mediated by a different set of effectors such as $\mathrm{Nbs} 1$ or Smc1 that regulate replication dynamics of damaged DNA independently of Chk2. In addition, in irradiated S and G2 cells, ATM coordinates the activation of Chk1 through the recruitment of ATR to the damaged chromatin, which, in turn, regulates IR-induced checkpoint responses. The S/G2 specificity of this pathway is reminiscent of the kinase activity of CDKs. In fact, a recent publication has shown that the use of a broad spectrum CDK inhibitor inhibits IR-induced Chk1 phosphorylation (23). However, it is still unclear how CDK activity is linked to the activation of this pathway. Regardless of the cell cycle specificity, our results demonstrate an active cross talk between ATM and ATR signaling pathways that cooperate in the molecular cascade that controls checkpoint signaling in postreplicative mammalian cells.

\section{MATERIALS AND METHODS}

Cell lines. Raji (CCL-86) and $\mathrm{ATR}^{\text {flox/- }}(\mathrm{CRL}-2780)$ human cell lines were purchased from the American Type Culture Collection. ATR wildtype (GM12072), ATR-Seckel (GM18367), ATM wild-type (GM00367), and AT (GM09607) cell lines were obtained from the Coriell Cell Repositories. Additional wild-type and AT lymphoblastoid lines were gifts of Y. Shiloh (Tel Aviv University, Tel Aviv, Israel). In the case of ATR ${ }^{\text {flox/- }}$ cells, biochemistry was performed $48-60 \mathrm{~h}$ after infection with adenovirus Cre. As reported previously (18), no detectable differences in cell cycle distribution were identified at these times after infection (unpublished data). 
Chromatin-bound fractionation and immunoblotting. The details of this protocol have been reported previously (19). In brief, $\sim 10^{7}$ cells were first resuspended for $5 \mathrm{~min}$ on ice in $150 \mu \mathrm{l}$ of fractionation buffer $(50 \mathrm{mM}$ Hepes, $\mathrm{pH} 7.5,150 \mathrm{mM} \mathrm{NaCl}$, and $1 \mathrm{mM}$ EDTA) containing $0.04 \%$ Nonidet P-40 supplemented with protease and phosphatase inhibitors. After centrifugation, the supernatant was collected (fraction I), and pellets containing all of the nuclei were washed twice with the same buffer. Nuclear pellets were further extracted for $40 \mathrm{~min}$ on ice with $150 \mu \mathrm{l}$ of fractionation buffer containing $0.5 \%$ Nonidet P-40. The extracts were clarified by centrifugation at $16,000 \mathrm{~g}$ for $15 \mathrm{~min}$ (chromatin-bound fraction). Aliquots of each fraction derived from equivalent cell numbers were separated on 4-12\% SDS-PAGE gels and blotted onto polyvinylidene difluoride membranes. Equal loading of fractions from treated and untreated samples was verified by immunoblotting against the nuclear factor ORC2 (a gift of J. Mendez, Spanish National Cancer Center, Madrid, Spain). ATM-1981 ${ }^{\mathrm{P}}$ (Rockland Immunochemicals, Inc.), ATM (Novus Biologicals), ATR (Serotec), Chk2 (Upstate Biotechnology), Chk1 (Novocastra), $\beta$-actin (Sigma-Aldrich), Chk2-T68 ${ }^{\mathrm{P}}$, and Chk1-S345 ${ }^{\mathrm{P}}$ (Cell Signaling Technology) primary antibodies were used in this study.

Flow cytometry of DNA damage-induced phosphorylation events. Our protocol is a variation of a recent method used to evaluate phosphoepitope status by flow cytometry (22). $5 \times 10^{5}$ cells were fixed by adding formaldehyde directly into the culture medium to obtain a final concentration of $1.5 \%$. Cells were incubated in fixative for $10 \mathrm{~min}$ at room temperature and collected by centrifugation. They were then permeabilized by resuspending with vigorous vortexing in $500 \mu \mathrm{l}$ of ice-cold $\mathrm{MeOH}$ and incubated at $4^{\circ} \mathrm{C}$ for $10 \mathrm{~min}$. Cells were washed twice in staining media (PBS containing 1\% BSA), resuspended in staining media containing Chk2-T68 ${ }^{\mathrm{P}}$ or Chk1-S345 ${ }^{\mathrm{P}}$ primary antibodies (Cell Signaling Technology), and incubated for $1 \mathrm{~h}$ at room temperature. After washing and repeating the process with AlexaFluor488-conjugated secondary antibodies (Invitrogen), the cells were finally resuspended in staining media containing $5 \mu \mathrm{g} / \mathrm{ml}$ propidium iodide and incubated for $20 \mathrm{~min}$ at $37^{\circ} \mathrm{C}$. Flow cytometry was evaluated in a FACScanner (Beckton Dickinson).

Immunocytochemistry. Cells were seeded onto an $18-\mathrm{mm}^{2}$ coverslip and were exposed to DNA damaging conditions $24 \mathrm{~h}$ later. 45 (IR) or 120 (HU) min after the exposure, cells were processed with a previously reported in situ fractionation technique (20) and stained with ATR (Santa Cruz Biotechnology, Inc.), $\boldsymbol{\gamma}$-H2AX (Upstate Biotechnology), and RPA (Rockland Immunochemicals, Inc.) recognizing primary antibodies. AlexaFluor488and 568-conjugated secondary antibodies were obtained from Invitrogen. DNA was counterstained with DAPI.

Online supplemental material. Fig. S1 shows the specificity of wortmannin-mediated inhibition of ATM. Fig. S2 shows the cell cycle distribution analysis of irradiated Raji cells in the presence or absence of wortmannin $45 \mathrm{~min}$ after exposure to the radiation source. Where indicated in these figures, ATM activity was inhibited by a previous $15-$ min treatment with $20 \mu \mathrm{M}$ wortmannin. Online supplemental material is available at http:// www.jem.org/cgi/content/full/jem.20051923/DC1.

The authors wish to thank Juan Mendez for the gift of ORC2 antibodies and for helpful discussions throughout the study.

This work is supported by Ramón y Cajal contracts from the Spanish Ministry of Science and Education to O. Fernandez-Capetillo and M. Murga and by Fundación La Caixa, Swiss Bridge, and Centro Nacional de Investigaciones Oncológicas Fundación Caja Madrid grants to 0. Fernandez-Capetillo.

The authors have no conflicting financial interests.

Submitted: 26 September 2005

Accepted: 12 January 2006

\section{REFERENCES}

1. Elledge, S.J. 1996. Cell cycle checkpoints: preventing an identity crisis. Science. 274:1664-1672.
2. Rouse, J., and S.P. Jackson. 2002. Interfaces between the detection, signaling, and repair of DNA damage. Science. 297:547-551.

3. Kastan, M.B., and J. Bartek. 2004. Cell-cycle checkpoints and cancer. Nature. 432:316-323.

4. Bartkova, J., Z. Horejsi, K. Koed, A. Kramer, F. Tort, K. Zieger, P. Guldberg, M. Sehested, J.M. Nesland, C. Lukas, et al. 2005. DNA damage response as a candidate anti-cancer barrier in early human tumorigenesis. Nature. 434:864-870.

5. Gorgoulis, V.G., L.V. Vassiliou, P. Karakaidos, P. Zacharatos, A. Kotsinas, T. Liloglou, M. Venere, R.A. Ditullio Jr., N.G. Kastrinakis, B. Levy, et al. 2005. Activation of the DNA damage checkpoint and genomic instability in human precancerous lesions. Nature. 434:907-913.

6. Abraham, R.T. 2004. PI 3-kinase related kinases: 'big' players in stressinduced signaling pathways. DNA Repair (Amst.). 3:883-887.

7. Collis, S.J., T.L. DeWeese, P.A. Jeggo, and A.R. Parker. 2005. The life and death of DNA-PK. Oncogene. 24:949-961.

8. Abraham, R.T. 2001. Cell cycle checkpoint signaling through the ATM and ATR kinases. Genes Dev. 15:2177-2196.

9. Fernandez-Capetillo, O., H.T. Chen, A. Celeste, I. Ward, P.J. Romanienko, J.C. Morales, K. Naka, Z. Xia, R.D. Camerini-Otero, N. Motoyama, et al. 2002. DNA damage-induced G2-M checkpoint activation by histone H2AX and 53BP 1. Nat. Cell Biol. 4:993-997.

10. Takai, H., K. Naka, Y. Okada, M. Watanabe, N. Harada, S. Saito, C.W. Anderson, E. Appella, M. Nakanishi, H. Suzuki, et al. 2002. Chk2deficient mice exhibit radioresistance and defective p53-mediated transcription. EMBO J. 21:5195-5205.

11. Brown, E.J., and D. Baltimore. 2003. Essential and dispensable roles of ATR in cell cycle arrest and genome maintenance. Genes Dev. 17:615-628.

12. Liu, Q., S. Guntuku, X.S. Cui, S. Matsuoka, D. Cortez, K. Tamai, G. Luo, S. Carattini-Rivera, F. DeMayo, A. Bradley, et al. 2000. Chk1 is an essential kinase that is regulated by Atr and required for the $G(2) / M$ DNA damage checkpoint. Genes Dev. 14:1448-1459.

13. Sarkaria, J.N., R.S. Tibbetts, E.C. Busby, A.P. Kennedy, D.E. Hill, and R.T. Abraham. 1998. Inhibition of phosphoinositide 3-kinase related kinases by the radiosensitizing agent wortmannin. Cancer Res. 58:4375-4382.

14. Ward, I.M., X. Wu, and J. Chen. 2001. Threonine 68 of Chk2 is phosphorylated at sites of DNA strand breaks. J. Biol. Chem. 276:47755-47758.

15. Melchionna, R., X.B. Chen, A. Blasina, and C.H. McGowan. 2000. Threonine 68 is required for radiation-induced phosphorylation and activation of Cds 1. Nat. Cell Biol. 2:762-765.

16. Bakkenist, C.J., and M.B. Kastan. 2003. DNA damage activates ATM through intermolecular autophosphorylation and dimer dissociation. Nature. 421:499-506.

17. O’Driscoll, M., V.L. Ruiz-Perez, C.G. Woods, P.A. Jeggo, and J.A. Goodship. 2003. A splicing mutation affecting expression of ataxiatelangiectasia and Rad3-related protein (ATR) results in Seckel syndrome. Nat. Genet. 33:497-501.

18. Cortez, D., S. Guntuku, J. Qin, and S.J. Elledge. 2001. ATR and ATRIP: partners in checkpoint signaling. Science. 294:1713-1716.

19. Andegeko, Y., L. Moyal, L. Mittelman, I. Tsarfaty, Y. Shiloh, and G. Rotman. 2001. Nuclear retention of ATM at sites of DNA double strand breaks. J. Biol. Chem. 276:38224-38230.

20. Mirzoeva, O.K., and J.H. Petrini. 2001. DNA damage-dependent nuclear dynamics of the Mre11 complex. Mol. Cell. Biol. 21:281-288.

21. Gatei, M., K. Sloper, C. Sorensen, R. Syljuasen, J. Falck, K. Hobson, K. Savage, J. Lukas, B.B. Zhou, J. Bartek, and K.K. Khanna. 2003. Ataxia-telangiectasia-mutated (ATM) and NBS1-dependent phosphorylation of Chk1 on Ser-317 in response to ionizing radiation. J. Biol. Chem. 278:14806-14811.

22. Perez, O.D., P.O. Krutzik, and G.P. Nolan. 2004. Flow cytometric analysis of kinase signaling cascades. Methods Mol. Biol. 263:67-94.

23. Jazayeri, A., J. Falck, C. Lukas, J. Bartek, G.C. Smith, J. Lukas, and S.P. Jackson. 2005. ATM- and cell cycle-dependent regulation of ATR in response to DNA double-strand breaks. Nat. Cell Biol. 8:37-45. 